\title{
Monoketone analogs of curcumin, a new class of Fanconi anemia pathway inhibitors
}

\author{
Igor Landais ${ }^{1}$, Sanne Hiddingh ${ }^{1}$, Matthew McCarroll ${ }^{1}$, Chao Yang ${ }^{2}$, \\ Aiming Sun ${ }^{2}$, Mitchell S Turker ${ }^{3}$, James P Snyder ${ }^{2}$ and Maureen E Hoatlin*1
}

\author{
Address: ${ }^{1}$ Department of Biochemistry and Molecular Biology, Oregon Health and Science University, Portland, USA, ${ }^{2}$ Chemical Biology \\ Discovery Center, Emory University, Atlanta, USA and ${ }^{3}$ Center for Research on Occupational and Environmental Toxicology, Oregon Health and \\ Science University, Portland, USA \\ Email: Igor Landais - landaisi@ohsu.edu; Sanne Hiddingh - hiddingh@ohsu.edu; Matthew McCarroll - mccarrom@ohsu.edu; \\ Chao Yang - cyang26@emory.edu; Aiming Sun - asun2@emory.edu; Mitchell S Turker - turkerm@ohsu.edu; \\ James P Snyder - jsnyder@emory.edu; Maureen E Hoatlin* - hoatlinm@ohsu.edu \\ * Corresponding author
}

This article is available from: http://www.molecular-cancer.com/content/8/I//33

(C) 2009 Landais et al; licensee BioMed Central Ltd.

This is an Open Access article distributed under the terms of the Creative Commons Attribution License (http://creativecommons.org/licenses/by/2.0), which permits unrestricted use, distribution, and reproduction in any medium, provided the original work is properly cited.

\begin{abstract}
Background: The Fanconi anemia (FA) pathway is a multigene DNA damage response network implicated in the repair of DNA lesions that arise during replication or after exogenous DNA damage. The FA pathway displays synthetic lethal relationship with certain DNA repair genes such as ATM (Ataxia Telangectasia Mutated) that are frequently mutated in tumors. Thus, inhibition of FANCD2 monoubiquitylation (FANCD2-Ub), a key step in the FA pathway, might target tumor cells defective in ATM through synthetic lethal interaction. Curcumin was previously identified as a weak inhibitor of FANCD2-Ub. The aim of this study is to identify derivatives of curcumin with better activity and specificity.
\end{abstract}

Results: Using a replication-free assay in Xenopus extracts, we screened monoketone analogs of curcumin for inhibition of FANCD2-Ub and identified analog EF24 as a strong inhibitor. Mechanistic studies suggest that EF24 targets the FA pathway through inhibition of the NF-kB pathway kinase IKK. In HeLa cells, nanomolar concentrations of EF24 inhibited hydroxyurea (HU)-induced FANCD2-Ub and foci in a cell-cycle independent manner. Survival assays revealed that EF24 specifically sensitizes FA-competent cells to the DNA crosslinking agent mitomycin C (MMC). In addition, in contrast with curcumin, ATM-deficient cells are twofold more sensitive to EF24 than matched wild-type cells, consistent with a synthetic lethal effect between FA pathway inhibition and ATM deficiency. An independent screen identified 4H-TTD, a compound structurally related to EF24 that displays similar activity in egg extracts and in cells.

Conclusions: These results suggest that monoketone analogs of curcumin are potent inhibitors of the FA pathway and constitute a promising new class of targeted anticancer compounds.

\section{Background}

Fanconi anemia (FA) is a multigene genetic disease characterized by developmental defects, early bone marrow failure and genomic instability leading to a high incidence of cancers [1]. At the molecular level, the FA pathway is a highly integrated DNA damage response network of pro- 
teins implicated in the repair of various DNA lesions and particularly DNA interstrand crosslinks [2,3]. The pathway is composed of a core complex of at least 10 proteins (including FANCA, B, C, E, F, G, L, M, FAAP24 and FAAP100) that function as an E3 ubiquitin ligase for the monoubiquitylation and activation of FANCD2 and FANCI [3]. Downstream proteins such as FANCD1/ BRCA2, FANCJ/BRIP1 and FANCN/PALB2 have been linked to elevated risk of breast and ovarian cancers [4]. However, although the FA pathway is well-defined biochemically, its precise roles in the DNA damage response remain obscure.

The FA pathway is a potential target in anticancer therapy either through chemosensitization of tumor cells to DNA crosslinking agents such as melphalan and cisplatin [5,6] or by exploiting synthetic lethal interactions. Two genes have a synthetic lethal relationship if mutants for either gene are viable but the double mutation is lethal [7]. Targeting this particular type of genetic interaction in tumors is currently the subject of intense development due to the promising results of clinical trials using PARP inhibitors in BRCA1/2-deficient breast tumors [8,9]. High-throughput screens to identify genes displaying synthetic lethal interaction with genes frequently impaired in tumors are demonstrating the potential for discovering functional dependencies created by oncogenic mutations that may enable therapeutic intervention for cancers with "undruggable" genetic alterations such as RAS [10,11]. With regard to FA, D'Andrea and coworkers identified a set of DNA damage response genes required for the survival of FA-deficient cells including ATM (Ataxia Telangectasia Mutated)[12]. ATM is a major kinase involved in the sensing and repair of DNA double-strand breaks by homologous recombination [13]. Germline mutations in this gene cause the Ataxia Telangectasia cancer susceptibility syndrome [14], and ATM deficiencies (mutations or lack of expression) are also frequent in sporadic hematological malignancies such as chronic lymphocytic leukemia [15] and mantle cell lymphoma [16]. Because deficiency in the FA pathway is not lethal [2], specific inhibitors are expected to display low toxicity toward normal cells but kill tumor cells deficient in ATM or other genes with synthetic lethal relationships to the FA pathway.

A cell-based screen for inhibitors of FANCD2 monoubiquitylation (FANCD2-Ub) recently identified curcumin [5], a phytochemical with anticancer properties that have been linked to a variety of mechanisms including apoptosis through the NFKB pathway [17]. Efforts to develop curcumin analogs with improved solubility, stability and activity have led to the generation of a series of monoketone derivatives including EF24, a strong candidate for further drug development in cancer therapy [18-22]. We evaluated these curcumin analogs in a cell-free assay that uses Xenopus egg extracts to uncouple FANCD2-Ub from ongoing replication $[6,23-26]$. The most active compounds were subsequently tested in mammalian cells for FA pathway inhibition and synthetic lethal interactions.

\section{Results \\ Inhibition of xFANCD2-Ub by monoketone analogs of curcumin in Xenopus extracts}

A series of monoketone analogs of curcumin [18] was evaluated in Xenopus egg extracts where DNA substrateinduced XFANCD2-Ub is used as a readout of FA pathway performance $[6,23,24]$. Phosphorylation of MRE11 (MRE11-P), a member of the MRN DNA damage repair pathway $[27,28]$ was monitored to assess the cross-specificity of the compounds. For each compound, $\mathrm{IC}_{50}$ values were determined from serial dilution experiments and densitometry analysis of immunoblots. Five analogs were at least 10 fold more active than curcumin both for inhibition of xFANCD2-Ub and xMRE11-P (Fig. 1A). Structure-activity relationship (SAR) analysis (Fig. 1B) suggested that a pyridine terminal aromatic ring and a nitrogen substitution in the central ring (analogs EF24, EF31, AS153-4, AS153-5) were important for activity. Accessibility of the lone electron pair on the nitrogen atom within the ring is crucial since movement of the basic nitrogen outside the ring (0810-117) or masking by a butoxycarbonyl (Boc) group (0616-104) decreases compound activity to non-detectable levels in Xenopus extracts. Replacement of the central ring nitrogen with sulfur while retaining the pyridine rings (0821-120A) drops the activity only slightly, whereas the same compound with an oxygen in the central ring was only weakly active (08223 ). None of the other combinations displayed a detectable activity in Xenopus extracts. Phosphorylation of other proteins involved in DNA damage response $\left(\mathrm{RPA}_{32}[29]\right.$ and H2AX, Fig. 1C) was not inhibited by the curcumin analogs.

\section{Mechanism of xFANCD2-Ub inhibition by EF24}

To explore potential mechanisms by which curcumin analogs inhibit the FA pathway, we evaluated the integrity of the FA core complex in Xenopus extracts containing EF24, one of the most active analogs. In human cells, hFANCD2-Ub is reduced when FA core complex proteins are defective or absent. However we found that the integrity of the core complex was unchanged in the presence or absence of EF24 (Additional File 1 - Fig. S1). Next, we found that EF24 did not detectably interfere with the recruitment of the core complex to DNA, a crucial step for xFANCD2 activation in Xenopus extracts (data not shown, [24]).

The proteasome is required for the monoubiquitylation of xFANCD2 in cells [30]. As curcumin has proteasome inhibiton activities [31], we tested whether EF24 inhibits 
A

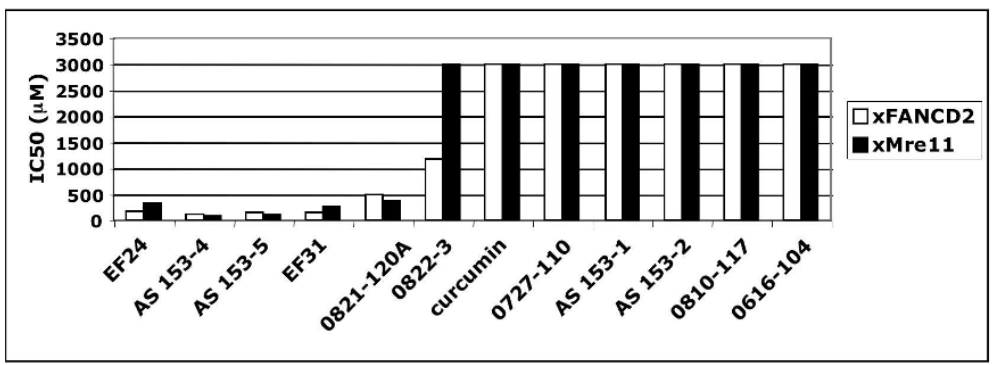

C

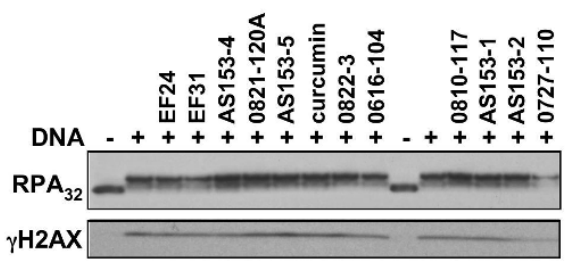

B
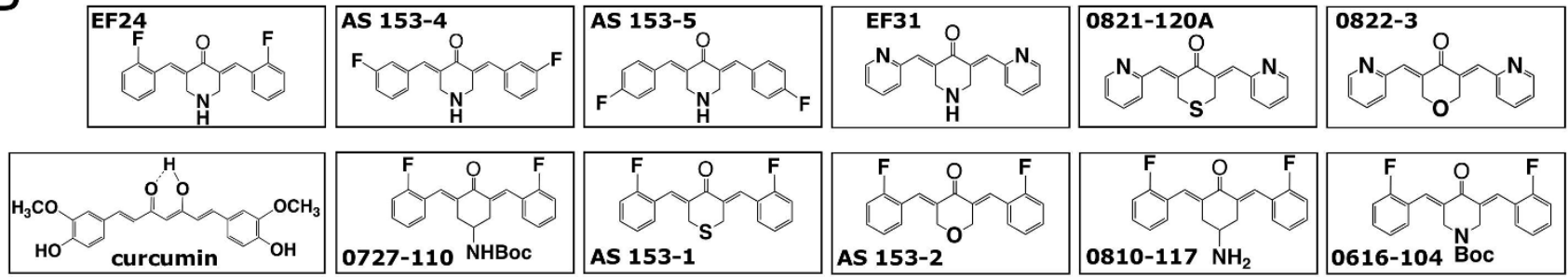

Figure I

Curcumin analogs efficiently inhibit xFANCD2 monoubiquitylation in Xenopus extracts. A) Inhibition of

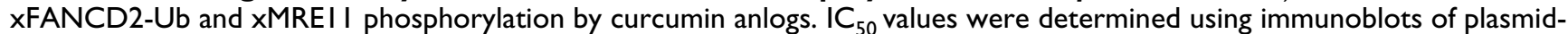
activated extracts treated with a range of concentrations for each compound $(0,10,25,60,150,400,1000,3000 \mu \mathrm{M})$. A representative experiment is shown (out of 3 repeats). B) Structure of curcumin and monoketone analogs. C) Curcumin analogs do not affect $\mathrm{RPA}_{32}$ and $\mathrm{H} 2 \mathrm{AX}$ phosphorylation. Activated extracts were treated with I mM each compound and phosphorylation of $\mathrm{RPA}_{32}$ and $\mathrm{H} 2 \mathrm{AX}$ monitored by immunoblot.

xFANCD2-Ub by this mechanism. Two proteasome activities (caspase-like and chymotrypsin-like) along with xFANCD2-Ub status were monitored in activated Xenopus extracts treated with various compounds (Fig. 2A). MG132 (a potent and specific proteasome inhibitor) and curcumin efficiently inhibited both proteasome activities but had only a weak activity on xFANCD2-Ub. By contrast, EF24 was 20-fold less active than curcumin for proteasome inhibition while displaying a strong activity against xFANCD2-Ub. Finally, the 0810-117 analog had weak inhibitory activities against both the proteasome and XFANCD2-Ub. To confirm the result obtained with EF24, we further tested EF31 and AS153-4, two close analogs that display a strong activity against xFANCD2-Ub (See Fig. 1A). Similar to EF24, both were 20-fold less active than curcumin for proteasome inhibition activity (Fig. 2B). Taken together, these results demonstrate that in Xenopus extracts EF24 and other monoketone analogs of curcumin do not inhibit the FA pathway by inhibiting the proteasome.

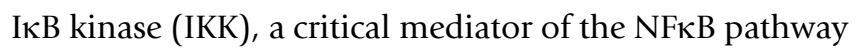
and the cellular response to stress, has recently been identified as an important target of EF24 for its apoptosis- mediated toxicity in cancer cells [32]. Interestingly, IKK has been suggested to play a role in the activation of the FA pathway [33]. We tested whether EF24 inhibits IKK activity in Xenopus extracts by monitoring the accumula-

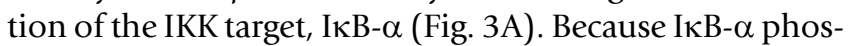
phorylation by IKK leads to its degradation by the ubiquitin-proteasome pathway, inhibition of IKK results in the accumulation of IкB- $\alpha$. For this experiment, we used extracts prepared without cycloheximide to allow for de novo translation of IKB- $\alpha$. We found that the level of IкB- $\alpha$ increased upon treatment of extracts with EF24, demonstrating that EF24 inhibits IKK in Xenopus extracts. Next, we reasoned that if EF24 inhibits xFANCD2-Ub through inhibition of IKK, a specific IKK inhibitor should inhibit XFANCD2-Ub. After treatment of Xenopus extracts with increasing concentrations of BMS-345541, a specific and potent IKK inhibitor [34], xFANCD2-Ub was inhibited in a dose-dependent manner (Fig. 3B). Similar to EF24 treatment, MRE11 phosphorylation was inhibited along with xFANCD2-Ub. EF24 was 10-15 times more active than BMS-345541 for xFANCD2-Ub inhibition $\left(\mathrm{EC}_{50}: 60 \mu \mathrm{M}\right.$ vs. $\left.970 \mu \mathrm{M}\right)$ and IкB- $\alpha$ stabilization $\left(\mathrm{EC}_{50}\right.$ : $30 \mu \mathrm{M}$ vs. $280 \mu \mathrm{M}$ ). Interestingly, EF24 and BMS-345541 concentrations required for XFANCD2-Ub inhibition were 

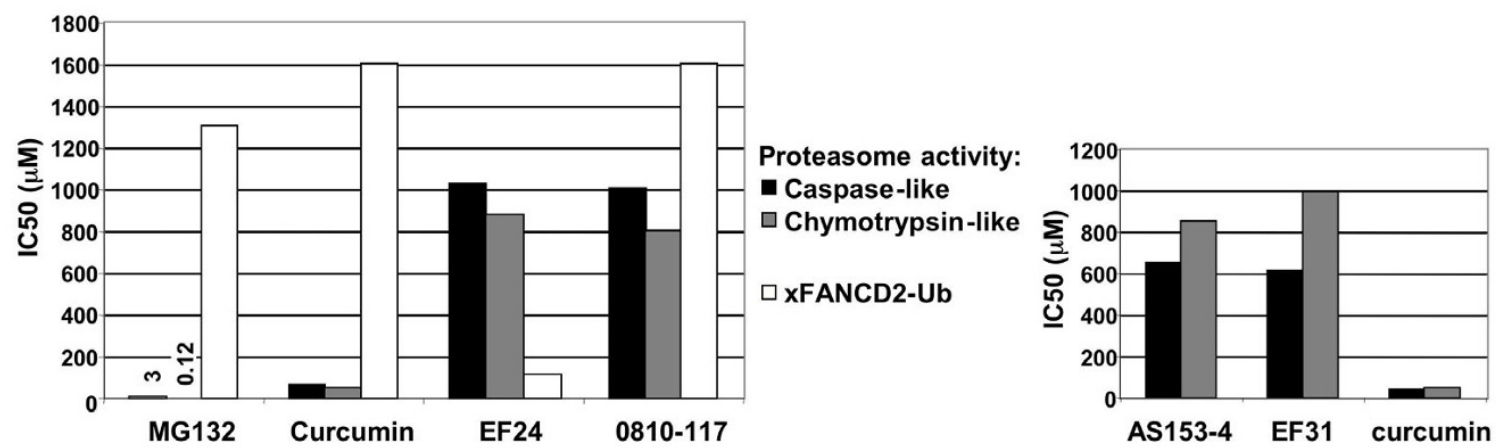

\section{Figure 2}

Proteasome inhibition activity of EF24 and related curcumin analogs in Xenopus extracts. A) Inhibition of caspaseand chymotrypsin-like proteasome activities and xFANCD2-Ub by MGI32, curcumin and curcumin analogs. Proteasome activities and xFANCD2-Ub were monitored using fluorogenic probes and immunoblotting, respectively. $I C_{50}$ values were determined for each compound using a range of concentrations and represented in histogram graphs. For MGI32, numbers above caspase-like and chymotrypsin-like histograms indicate the actual $\mathrm{IC}_{50}$ values. A representative experiment is shown (out of 3 repeats). B) Inhibition of proteasome activities by EF3I and ASI53-4 compared to curcumin.

higher than that required for IкB- $\alpha$ stabilization (EF24 $\mathrm{EC}_{50}: 60 \mu \mathrm{M}$ vs. $30 \mu \mathrm{M}$; BMS-345541 $\mathrm{EC}_{50}: 970 \mu \mathrm{M}$ vs. $280 \mu \mathrm{M}$, Fig. 3A and 3B), suggesting that the kinase activity of IKK is not crucial for xFANCD2-Ub in Xenopus extracts. To investigate this idea further, we tested whether EF24 inhibition of xFANCD2-Ub is dependent on phosphorylation (Fig. 3C). Treatment of extracts with tautomycin, a PP1/PP2A phosphatase inhibitor, resulted in the accumulation of XMRE11-P even in the presence of EF24 but had no detectable effect on xFANCD2-Ub. Treatment with caffeine, a PIKK kinase inhibitor, significantly inhibited XMRE11-P (compare lane 12 and lanes 15, 18) but co-treatment with EF24 did not alter the pattern of xFANCD2-Ub inhibition (compare lanes 12-14 and 15$17,18-20)$. Since caffeine is not a general kinase inhibitor, we also used shrimp alkaline phosphatase (SAP) to dephosphorylate proteins non-specifically in extracts. Treatment with $0.1 \mathrm{u} / \mu \mathrm{l}$ SAP significantly reduced xMRE11-P levels (compare lanes 12 and 24) but did not affect EF24-dependent inhibition of xFANCD2-Ub (compare lanes 12-14 and 24-26). Taken together, these results suggest that EF24 might inhibit the FA pathway by targeting IKK through a mechanism that does not involve inhibition of IKK kinase activity.

\section{EF24 inhibits hFANCD2-Ub and hFANCD2 foci in HeLa cells}

We next determined whether EF24 could inhibit hFANCD2-Ub in human cells. Curcumin was tested in parallel to assess whether activity difference observed in extracts are mirrored in cells. HeLa cells were co-treated with increasing concentrations of compounds and hydroxyurea (HU), a standard method to stimulate hFANCD2-Ub (Fig. 4A, lanes 1-2, 7-8, [30]). Immunoblot and densitometry analysis revealed that EF24 is 40-fold more active than curcumin for hFANCD2-Ub inhibition ( $\mathrm{IC}_{50}: 0.39 \mu \mathrm{M}$ vs. $15 \mu \mathrm{M}$ ), in line with the stronger activity of EF24 observed in extracts.

As the activity of the FA pathway is cell cycle dependent, we monitored the effect of HU and EF24 on the cell cycle by FACS analysis. We found no significant change in the cell cycle profile of cells treated with HU in combination with EF24 compared to that of untreated cells (Additional File 1 - Fig. S2), suggesting that EF24 does not inhibit hFANCD2-Ub through perturbation of the cell cycle.

To confirm inhibition of the FA pathway using another readout, we monitored the effect of EF24 on HU-induced hFANCD2 foci in HeLa cells (Fig. 4B) [29]. IC I0 $_{50}$ values for inhibition of foci formation were in the same range as $\mathrm{IC}_{50}$ values for hFANCD2-Ub inhibition, an expected result since hFANCD2-Ub but not the unmodified hFANCD2 is competent to form foci [35].

EF24 sensitizes FA-competent cells to mitomycin C (MMC) We tested whether EF24 is a specific inhibitor of the FA pathway by comparing its effect on a FANCA-deficient cell line (HSC72OT) and its complemented, wild-type-like counterpart (HSC72OT+A) [36] in the presence of the DNA crosslinking agent MMC. If EF24 specifically targets the FA pathway, it should sensitize FA-competent cells to MMC but not FA-deficient cells. 

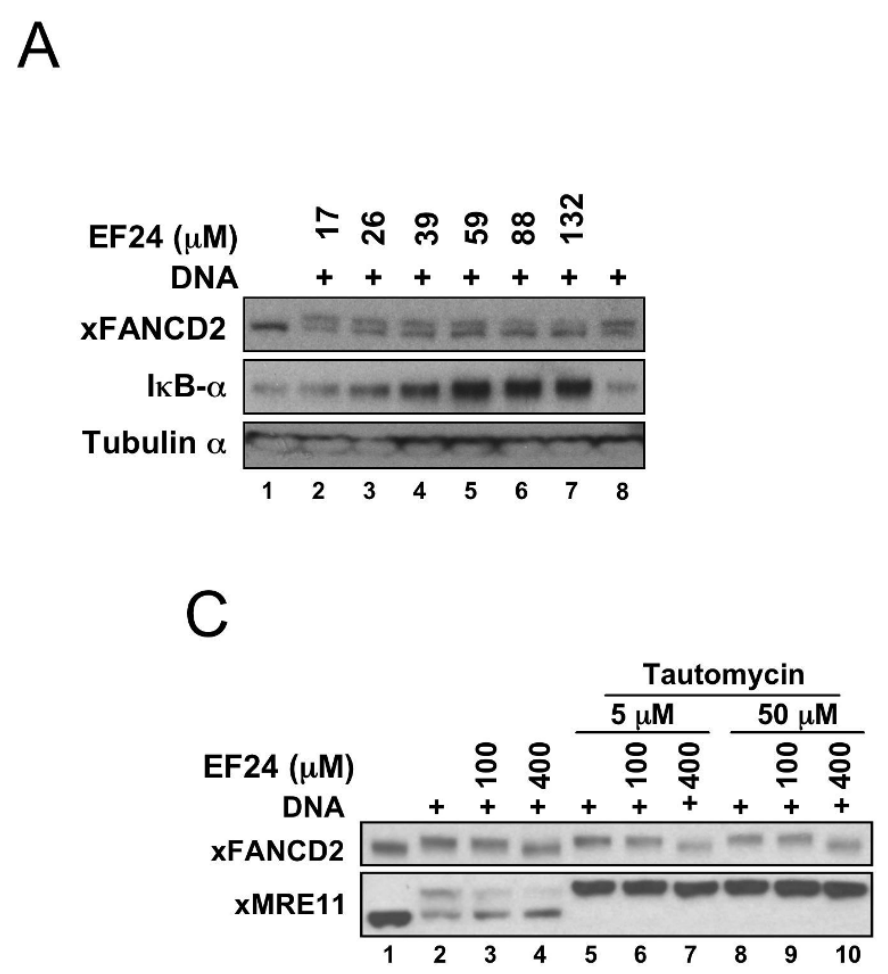

B<smiles>Cc1ccc2nc(NCCN)c3ncc(C)n3c2c1</smiles>

IKK inhibitor III $(\mu \mathrm{M})$

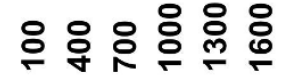

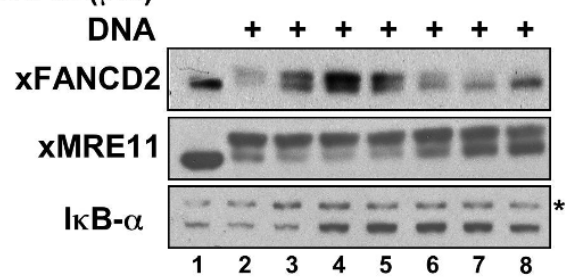

\section{Figure 3}

EF24 does not inhibit xFANCD2-Ub through modulation of a phosphorylation event, but IKK inhibition might play a role. A) EF24 inhibits the IKK kinase in Xenopus extracts. Extracts treated as indicated were analyzed by immunoblot using $x$ FANCD2, I $\mathrm{B}-\alpha$ and tubulin- $\alpha$ antibodies. I $\mathrm{K}$ - $\alpha$ protein level was used as readout for monitoring IKK inhibition. B) The specific IKK inhibitor compound BMS-34554I (IKK inhibitor III, Calbiochem) inhibits xFANCD2-Ub in extracts. Experiment was performed as in (A). The structure of BMS-34554I is shown. The star denotes a non-specific band used as loading control. C) EF24-dependent inhibition of xFANCD2-Ub is not affected by co-treatment with tautomycin (phosphatase inhibitor), caffeine (kinase inhibitor) and SAP (shrimp alkaline phosphatase). Extracts treated as indicated were analyzed by immunoblot using XFANCD2 and XMREII antibodies. XMREI I phosphorylation status was used to monitor the efficiency of tautomycin, caffeine and SAP treatments.

As expected, HSC72OT+A cells were more resistant to MMC compared to HSC72OT (Fig. 5A, compare solid line curves in left and right panels). $100 \mathrm{nM}$ EF24, a concentration that did not affect survival in either cell line (Fig. 5B) slightly reduced the survival of MMC-treated HSC72OT cells (Fig. 5A, left panel). By contrast, the same treatment induced a significant decrease of cell survival in HSC72OT+A cells (Fig. 5A, right panel), suggesting that EF24 sensitized HSC72OT+A cells to MMC by inhibiting the FA pathway.

\section{Sensitivity of ATM-deficient cells to EF24 suggests a synthetic lethal effect}

Kennedy et al [12] identified ATM in a screen for genes that display a synthetic lethal relationship with the FA pathway, suggesting that inhibition of the FA pathway could selectively kill ATM-deficient cells. To test this possibility, we compared the effects of curcumin and EF24 in an ATM KO mouse kidney cell line $\left(309_{\text {ATM ко }}\right)$ and its isogenic, wild type counterpart $\left(334_{\text {ATM WT }}\right)$. As expected from ATM deficiency, $309_{\mathrm{ATM}}$ кO cells were more sensitive to ionizing irradiation than $334_{\text {ATM WT }}$ (Additional File 1 Fig. S3). We next treated these cell lines with various concentrations of EF24 and measured their survival after 72 hrs. Fig. 6A shows that $309_{\text {ATM KO }}$ cells were twofold more sensitive to EF24 than $334_{\text {ATM WT }}$ cells $(2.6$ vs. $5.8 \mu \mathrm{M})$. In contrast, $309_{\text {ATM кO }}$ cells were slightly more resistant than $334_{\text {ATM WT }}$ to curcumin ( 23 vs. $17 \mu \mathrm{M}$, Fig. 6B), possibly due to the pleiotropic effects of curcumin masking the weak FA pathway inhibition activity. To rule out a general chemical sensitivity of the ATM KO cell line, we also tested the casein kinase II inhibitor DRB, a compound with no effect on the FA pathway in Xenopus extracts (data not shown). As expected, DRB displayed similar toxicity in both cell lines (Fig. 6C).

These results suggest that the toxicity of EF24 observed in ATM-deficient cells may be caused by a synthetic lethal 

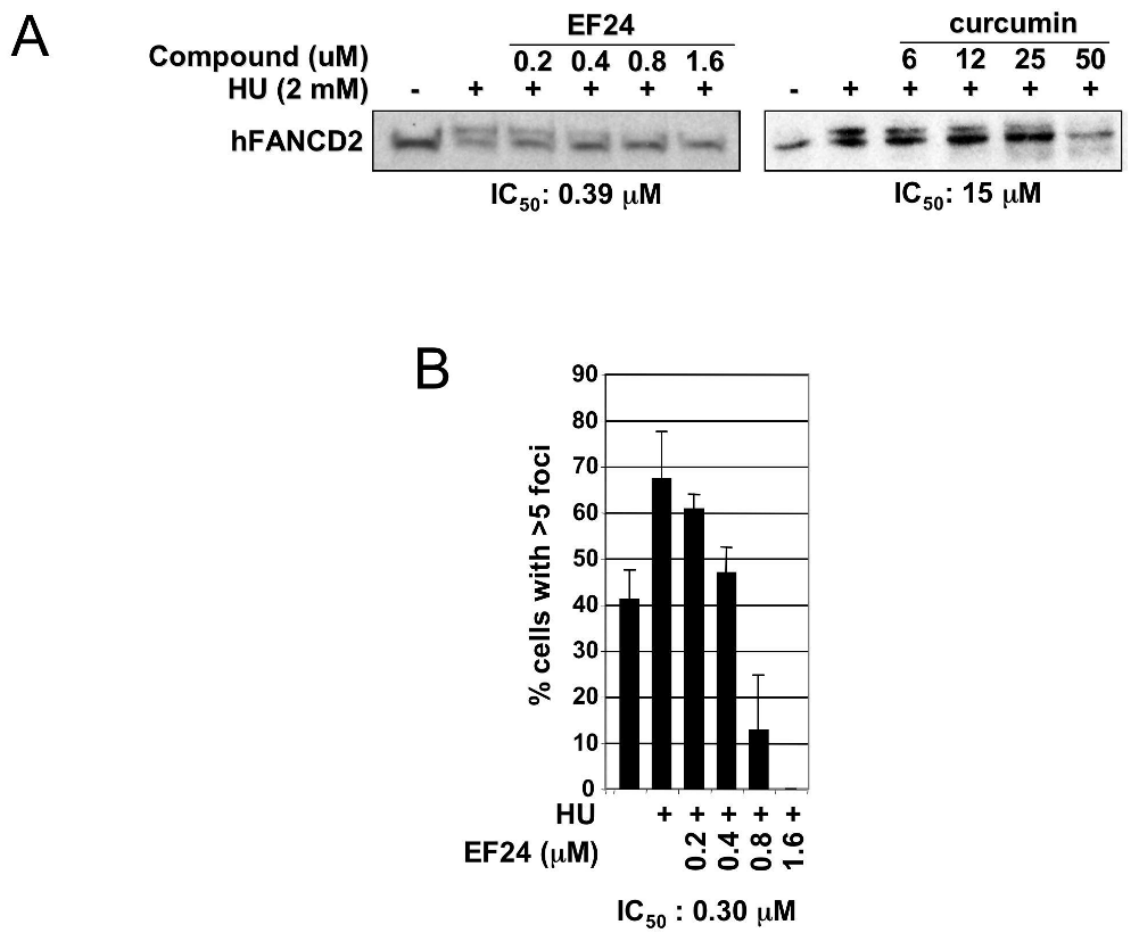

\section{Figure 4}

EF24 inhibits hFANCD2-Ub and foci formation in HeLa cells and is more active than curcumin. A) Hydroxyureainduced FANCD2-Ub in HeLa cells is inhibited by EF24 at lower concentrations than curcumin. hFANCD2-Ub was monitored by immunoblot and densitometry analysis to determine $I_{50}$. B) Hydroxyurea-induced hFANCD2 foci formation is inhibited by EF24. hFANCD2 foci were detected by immunofluorescence and the percentage of cells with more than 5 foci was determined for each treatment. Histograms represent the average of 3 experiments. Error bars represent standard deviation.

effect due to the inhibition of the FA pathway in the absence of ATM.

\section{H-TTD, a novel curcumin analog with properties similar to EF24}

We previously identified several small molecule inhibitors of the FA pathway using the Xenopus cell-free assay [6]. Structural analysis revealed that one of the compounds (E3 of the Challenge Set library, NCI) is a curcumin analog that resembles EF24 (Fig. 7A). The structure incorporates a sulfone moiety instead of an amine in the central ring and $p$-nitro substitution in the terminal phenyl rings instead of $o$-fluoro groups. Its chemical name, 4H-Thiopyran-4-one, tetrahydro-3,5-bis [(4-nitrophenyl)methylene]-,1,1-dioxide, was abbreviated to 4H-TTD for convenience. Comparison with curcumin and EF24 revealed that $4 \mathrm{H}-\mathrm{TTD}$ is slightly less active than EF24 for FA pathway inhibition in Xenopus extracts $\left(\mathrm{IC}_{50}: 250 \mu \mathrm{M}\right.$ vs. $150 \mu \mathrm{M}$, Fig. 7B). In HeLa cells, 4H-TTD inhibited HUinduced hFANCD2-Ub at sub-micromolar concentrations, similar to EF24 ( $\mathrm{IC}_{50}: 0.12 \mu \mathrm{M}$, Fig. 7C). We next tested 4H-TTD activity in the survival assay described in the previous paragraph (Fig. 6). Like EF24, 4H-TTD displayed more toxicity in $309_{\text {ATM KO }}$ cells, with a 3 -fold difference compared to WT cells ( 0.18 vs. $0.5 \mu \mathrm{M}$, Fig. $7 \mathrm{D})$. The overall cell toxicity was in the nanomolar range, about 10 times lower than EF24, suggesting that 4H-TTD has a general cellular toxicity greater than EF24.

These results underscore the use of Xenopus egg extracts as a useful screening strategy to rapidly identify inhibitors of the FA pathway and further validates this class of compounds as potent FA pathway inhibitors and potential anticancer agents for synthetic lethal relationship-based targeted therapy.

\section{Discussion}

DNA damage response pathways have recently been the target of considerable efforts in oncology research. This is due in part to the successful treatment of BRCA-deficient tumors with PARP1 inhibitors [8,9], which demonstrated that taking advantage of the synthetic lethal relationship between two DNA damage genes is a valid approach for the development of novel targeted therapies in oncology 
A
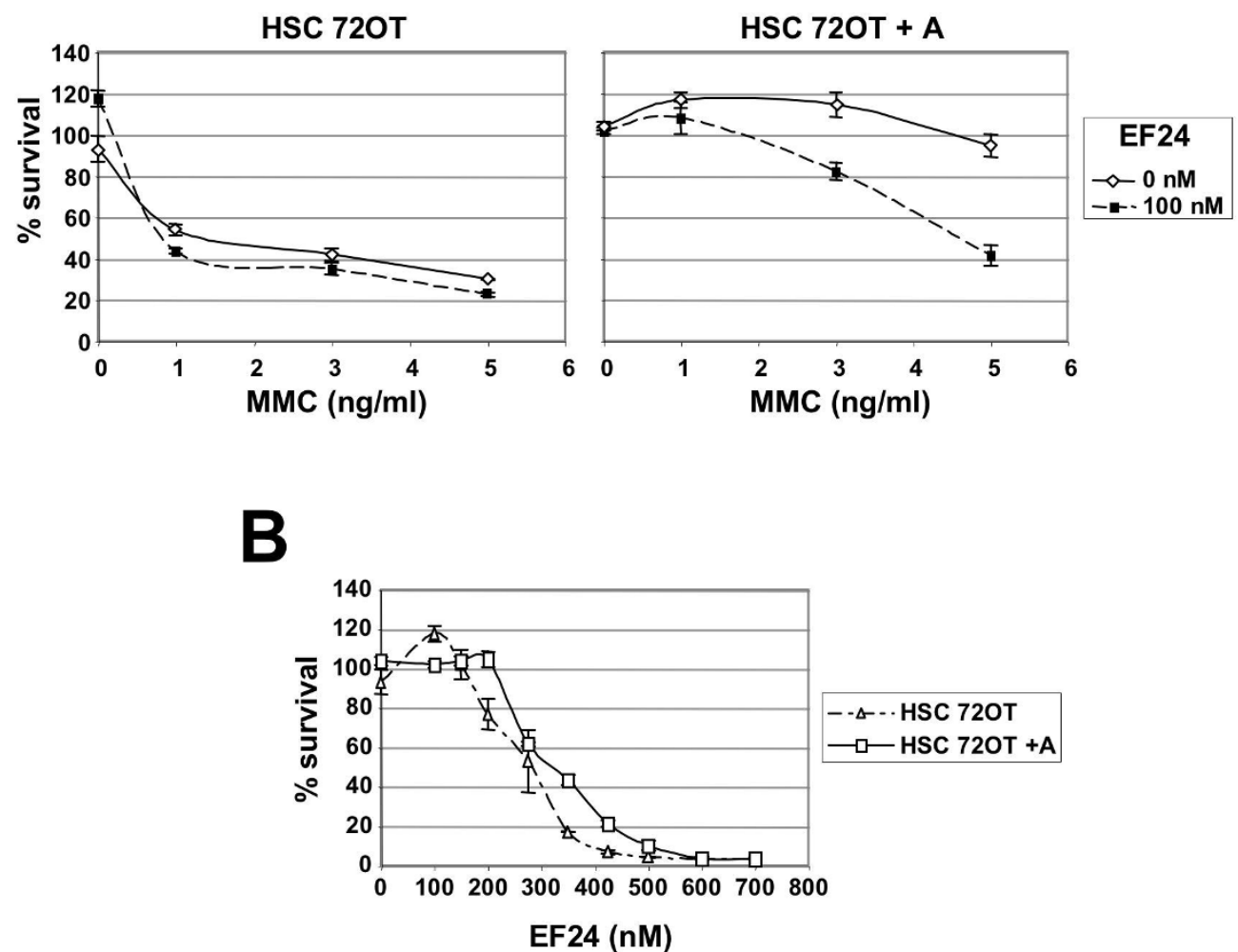

\section{Figure 5}

EF24 sensitizes the HSC 720T+A cell line to MMC but not its FA-deficient counterpart, HSC 72OT. A) Effect of the combination of MMC and EF24 treatment on the viability of HSC 72OT and HSC 72OT+A cells. HSC 72OT (Patientderived FANCA-deficient cell line) and HSC 72OT+A (FANCA-complemented isogenic cell line) were treated with various concentrations of MMC only (solid lines) or MMC + 100 nM EF24 (dashed lines). Cell viability was measured after 3 days by MTS assay. B) Similar experiment was performed using EF24 only.

[7]. Two parameters are important for a synthetic lethal interaction to be exploited therapeutically against cancers: first, functional deficiency of one of the genes has to be a causative event of the tumorigenesis. Impairment of DNA damage responses, which is strongly suspected to be one of the most common initial event during tumorigenesis [37], fits this requirement. The second condition is that the target of the therapeutic agent is not an essential gene, hence limiting toxicity and increasing the therapeutic window. Many DNA damage response pathways are partially redundant, explaining why one defect is usually well tolerated at least in the short term, e.g. PARP1 [7,38].

Synthetic lethal interactions between the FA pathway and other DNA damage response genes such as ATM, PARP1 and NBS1 have been identified recently [12], stimulating interest in determining if FA pathway inhibitors would selectively target tumor cells deficient in these genes. For instance, ATM mutations are highly prevalent (approx. $50 \%$ ) in mantle cell lymphoma patients [16], raising the possibility that these tumors might be selectively treated with a FA pathway inhibitor.

The first identified FA pathway inhibitor was curcumin [5]. To improve potency and specificity, we compared the FA pathway inhibition activity of a series of monoketone analogs of curcumin [18] in Xenopus extracts, and identified EF24 and 4 other compounds as potent FA pathway inhibitors. Similar to DDN and other inhibitors we previously identified [6] and data not shown), curcumin analogs displayed similar activity against MRE11 phosphorylation but did not inhibit $\mathrm{RPA}_{32}$ and $\mathrm{H} 2 \mathrm{AX}$ phosphorylation. This finding will be important practically for the design of a high-throughput assay, allowing rapid discrimination between specific FA pathway inhibitors and those that also inhibit MRE11 phosphorylation.

\section{Structure-activity relationships}

Structural similarity of EF24 and 4H-TTD confirmed that monoketone analogs of curcumin form a new class of FA 
A
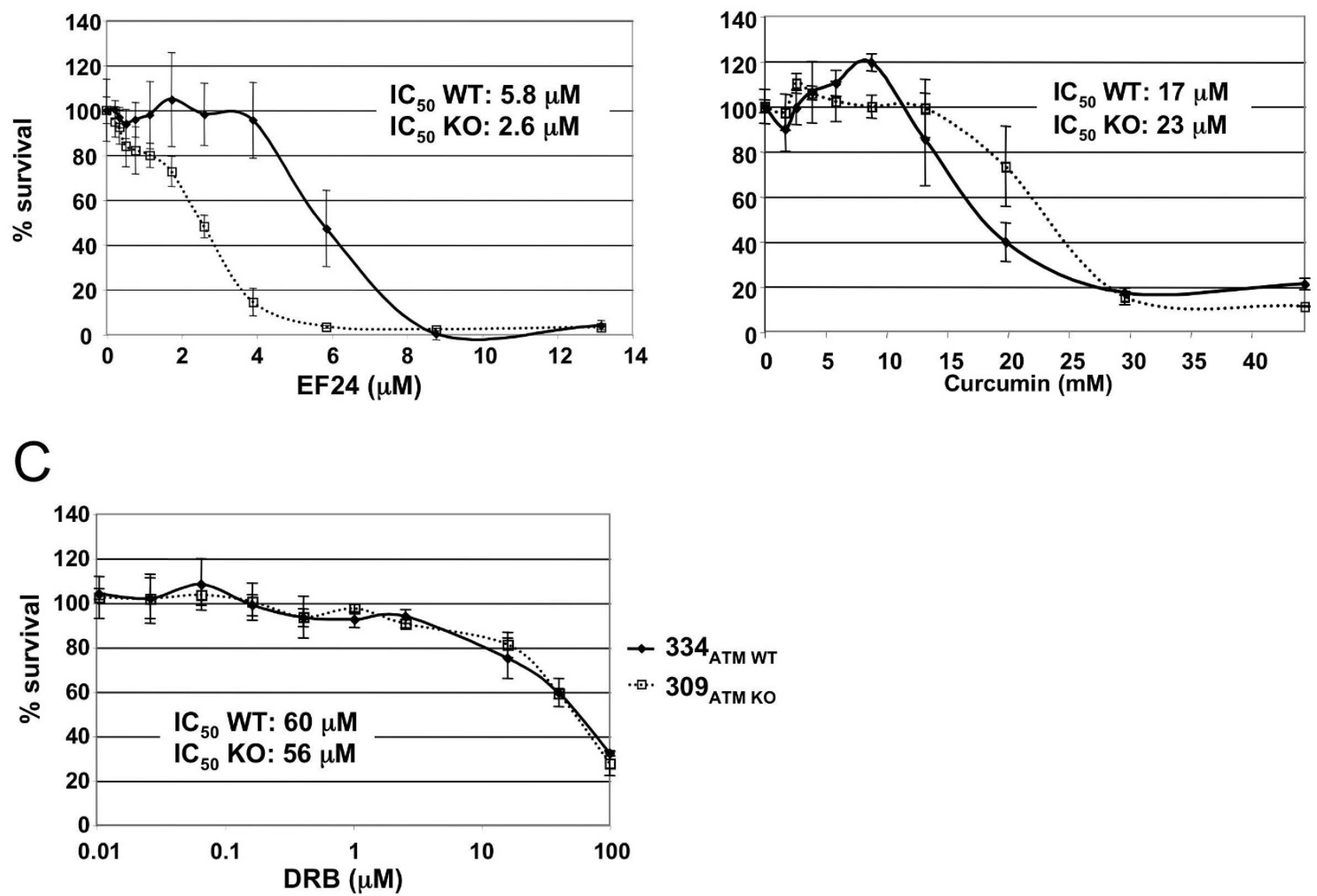

Figure 6

EF24 but not curcumin or DRB is more toxic to ATM-deficient mouse kidney cells than wild-type cells. A) $309_{\text {ATM KO }}$ and $334_{\text {ATM WT }}$ cells were treated with various concentrations of EF24. Cell viability was measured after 3 days by MTS assay. Each point represents the mean of 3 repeats. Error bars represent standard deviation. B) Similar experiment was performed using curcumin. C) Similar experiment was performed using DRB, a caseine kinase II inhibitor that does not inhibit the FA pathway in Xenopus extracts.

pathway inhibitors. Within the same structural template, heteroatom ring replacements and a subset of terminal aromatic ring substituents appear to account for the potency of a given compound. With respect to the central six-membered ketone for example, the piperidone derivatives (EF24, EF31, AS153-4, and AS153-5) are much more potent than the corresponding thio-pyran analog (AS1531 ). However, decorating the nitrogen of the piperidone with a strong electron withdrawing group that diminishes the basicity of the nitrogen, for instance the Boc group, causes complete loss of activity (0616-104). Switching from piperidone to pyran or cyclohexanone likewise decreases activity to undetectable levels in Xenopus extracts (AS153-2 and 0810-117). By contrast, the central sulfone of 4H-TTD restores activity perhaps by virtue of its unique ability to serve as a proton acceptor on both faces of the central ring. With respect to terminal ring substitution, the moieties that deliver the most potent analogs are F, OH and the pyridine nitrogen (Fig. 1) [18]. Aromatic nitro groups coupled with a central ring sulfone as represented by 4H-TTD (Fig. 7A) represents a novel combination. This emerging SAR provides a direction for further optimization. For example, it will be instructive to see if combining nitro groups with a piperidone ring or fluoro substituents with sulfone will either match or enhance the observed activities.

While exploring the mechanism of FA pathway inhibition, we found that EF24 is a weak proteasome inhibitor. The more effective proteasome inhibition by curcumin 


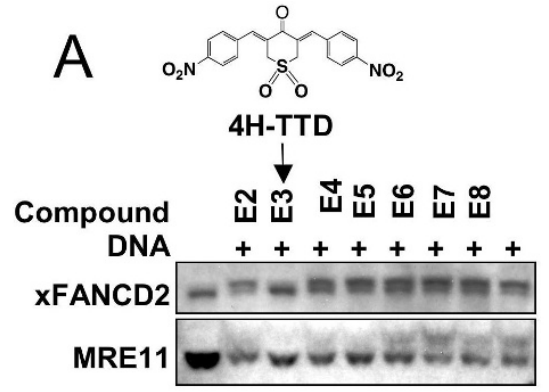

C

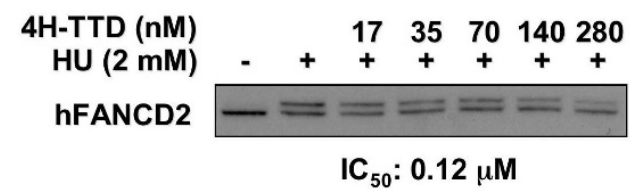

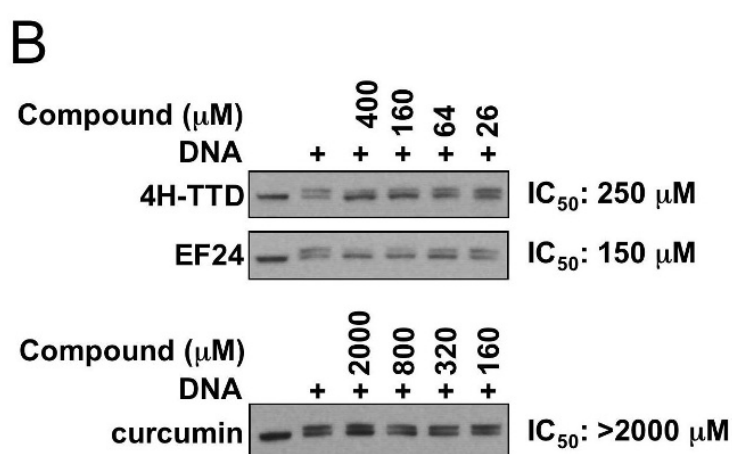

$\mathrm{D}$

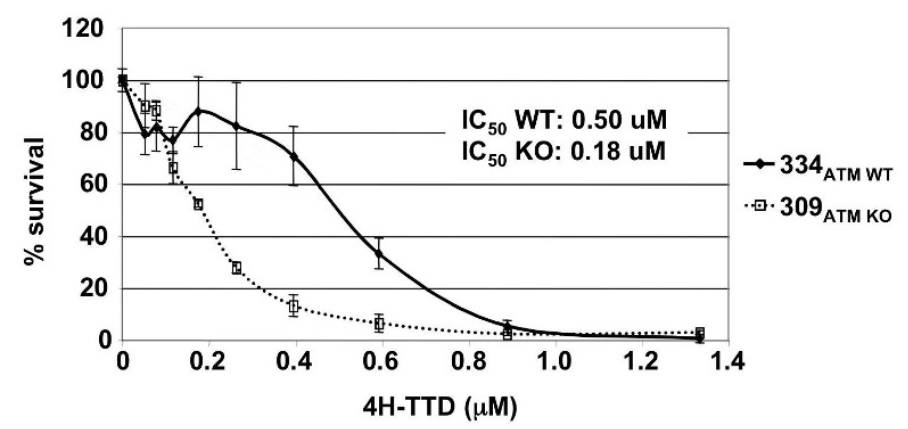

Figure 7

Monoketone analogs of curcumin form a new class of FA pathway inhibitors. A) Identification of $4 \mathrm{H}-\mathrm{TTD}$ in an independent screen for FA pathway inhibitors. Each compound of row $\mathrm{E}$ of the $\mathrm{NCl}$ Challenge Set library plate was tested at 0.5 $\mathrm{mM}$ in Xenopus extracts for inhibition of xFANCD2-ub and XMREI I-P. Arrow indicates the active compound. The chemical structure of $4 \mathrm{H}-\mathrm{TTD}$ is shown. B) Comparison of $x$ FANCD2-Ub inhibition activity of 4H-TTD, EF24 and curcumin in Xenopus extracts. xFANCD2-Ub IC I0 $_{50}$ were determined as in Fig. IA. C) 4H-TTD inhibits the FA pathway in HeLa cells. IC 50 was determined as in Fig. 4A. D) $4 \mathrm{H}$-TTD is more toxic to $309_{\text {ATM KO }}$ than $334_{\text {ATM WT }}$ cells.

(Fig. 2) may be due to structural features absent in the monocarbonyl analogs. Three prominent structural elements that differentiate the two chemical classes are the central rigidifying ring in the analogs, the presence of the central three-carbon unit bearing two oxygens and the phenolic oxygens in curcumin. The antioxidant properties of curcumin are generally attributed to the radical scavenging abilities of the phenolic OH groups [39] and the central 1,3-diketone functionality [40]. Thus, either radical scavenging or a change in molecular architecture or both may be the basis for curcumin's superior activity against the proteasome. This result implies that EF24 has a spectrum of activities narrower than curcumin.

\section{Inhibition of the FA pathway in cells}

With activity in the nanomolar range in cells, EF24 and 4 H-TTD are the most active FA pathway inhibitor identified so far $[5,6,30]$. As previously noted [6], EF24 and 4HTTD effective concentrations were 2 to 3 orders of magnitude lower in cells than in extracts, probably because of the strong quenching occurring in extracts due to high proteins and lipids content [41].

We next tested whether inhibition of the FA pathway by EF24 could be exploited to target cells using sensitization and synthetic lethal interaction approaches. Using a combination strategy [6], we found that a non-toxic dose of EF24 sensitized FA-competent cells to the DNA crosslinking agent MMC. EF24 failed to sensitize isogenic FA-deficient cells to MMC, suggesting that inhibition of the FA pathway rather than pleiotropic activity was responsible for the sensitization phenotype observed (Fig. 5). Hence EF24 might be an interesting lead as a chemosensitizer for DNA crosslinking agents such as MMC and platinium compounds.

Next, EF24 and 4H-TTD were used as single agents in congenic cell lines differing in their ATM status. We found that both compounds were significantly more toxic to ATM KO cells than WT cells. These results are consistent 
with a model where inhibition of the FA pathway by curcumin analogs triggered a synthetic lethal effect in ATMdeficient cells [12]. The failure of curcumin to display the same effect could be due to the fact that its weak activity against the FA pathway is masked by the activity toward numerous other targets, for instance the proteasome.

\section{Mechanism of FA pathway inhibition}

EF24 did not inhibit the FA pathway by well-known mechanisms such as disrupting the integrity of the FA core complex [42], impairement of recruitment of FA proteins to DNA substrates [24] or proteasome inhibition [30]. Surprisingly, proteasome inhibition did not affect xFANCD2-Ub in Xenopus extracts, suggesting that the proteasome is not involved in the basic activation of the FA pathway. Rather, it may be required in the more complex cellular environment to overcome the chromatin barrier or to respond to specific DNA lesions [30]. Importantly, this finding further highlights the use of the Xenopus extracts-based screening assay to identify compounds that are more likely to target the core FA pathway rather than general regulators. Underscoring this notion, monoubiquitylation of FANCD2 is also independent of phosphorylation in DNA-stimulated extracts (Fig. 3C[24]).
Interestingly, Aleo E. et al [43] identified 4H-TTD (called "G5" in their work) in a screen for compounds that trigger caspase activation in a BCL-2 dependent and caspase 9independent manner. 4H-TTD did not inhibit proteasome activity, confirming the data obtained with EF24 and related analogs. Instead, 4H-TTD exhibited ubiquitin isopeptidase/deubiquitinase (DUB) inhibition activity. Structural analysis further revealed a molecular determinant shared with known ubiquitin isopeptidase inhibitors [43] that is also present in EF24, raising the possibility that monoketone analogs of curcumin could inhibit FANCD2- Ub through a novel mechanism involving DUB inhibition independently of the proteasome.

During this study, we found that EF24 inhibits the phosphorylation of xMRE1 1 and XFANCM in Xenopus extracts (Fig. 1, 3 and Additional File 1 - Fig S1). Since both proteins are the target of the DNA damage signaling and checkpoint kinases ATM and ATR [28,44], we tested whether EF24 treatment affected the phosphorylation status of a classic ATR target, CHK1 [45]. Reprobing the EF24 blot shown in Fig. 7B with a phospho-CHK1-specific antibody revealed that CHK1-P was inhibited at the highest EF24 concentration tested $(400 \mathrm{mM}$, Additional File 1 -

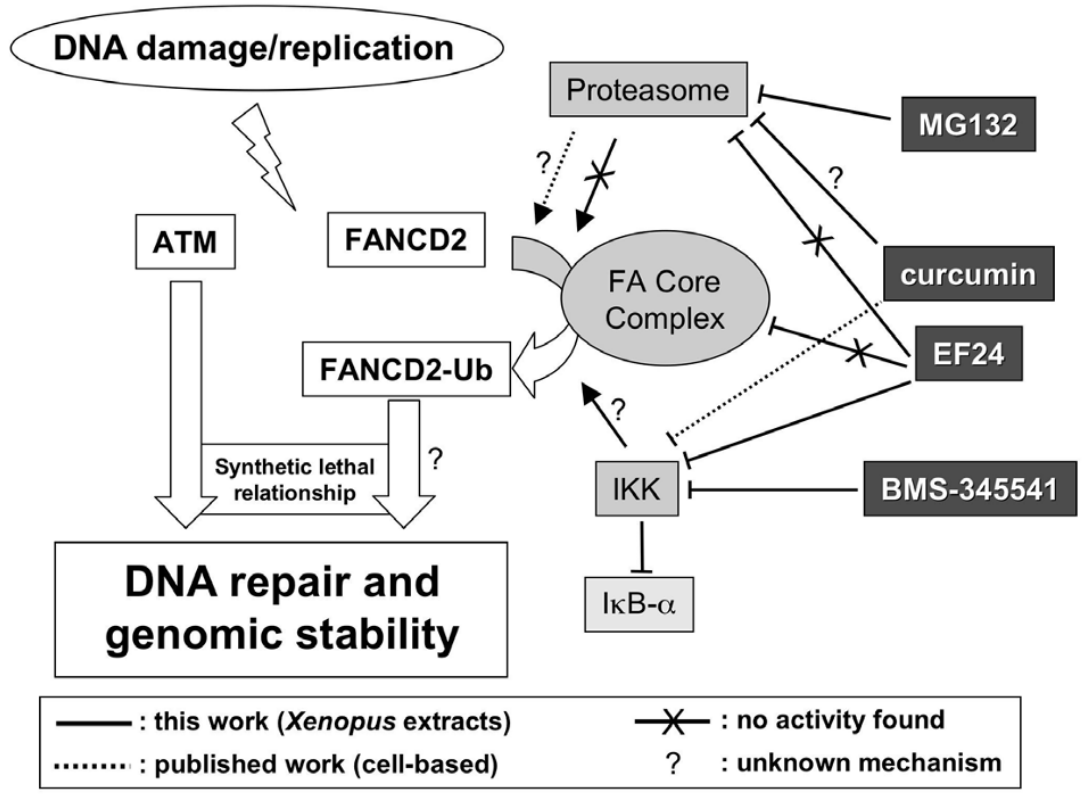

Figure 8

Summary of the mechanistic findings presented in this paper. The ATM and FA pathways (white boxes/arrows) are two DNA damage response pathways that display synthetic lethal relationship. Inhibition of the FA pathway may therefore be used as a targeted therapy to selectively kill ATM-deficient tumors. Several enzymatic complexes have been proposed to modulate the FA pathway (light grey boxes). Monitoring the effect of the compounds used in this study (dark grey boxes) toward those enzymatic activities suggests that (i) in contrast with cell-based assays, the proteasome is not required for FA pathway activation in Xenopus extracts, (ii) in contrast with curcumin, EF24 is a weak proteasome inhibitor, (iii) EF24 does not inhibit the FA pathway through disruption of the core complex, and (iv) curcumin, EF24 and BMS-34554I might inhibit the FA pathway through inhibition of IKK. 
Fig. S4), suggesting that this curcumin analog is a bona fide but weak ATR inhibitor. Since FANCD2 monoubiquitylation has been shown to be dependent on ATR both in mammalian cells $[46,47]$ and in replicating Xenopus extracts $[23,26,44]$, it was therefore tempting to hypothesize that EF24 inhibits FANCD2-Ub through ATR inhibition. However our lab has clearly demonstrated that FANCD2-Ub is not dependent on ATR in non-replicating, DNA substrate-stimulated extracts: chemical inhibition of ATR by caffeine or depletion of ATR and its essential partner ATRIP have no detectable effect on FANCD2-Ub in this setting (Fig. 3C and [24,44]). Taken together, these data suggest that EF24 might be a weak ATR inhibitor, but this activity does not account for FANCD2-Ub inhibition in non-replicating extracts.

An alternative hypothesis emerged from the fact that one of the cellular targets of curcumin and EF24 is the I $\mathrm{KB}$ kinase complex (IKK), a major component of the NF- $\kappa B$ pathway. Otsuki et al [33] showed that IKK interacts with the FA core complex, raising the possibility that EF24 might inhibit the FA pathway by targeting IKK. This hypothesis is in line with the fact that curcumin has a weaker inhibitory activity than EF24 toward both IKK and xFANCD2-Ub (Fig. 1, Fig. 4 and [32]). Interestingly, we found that the specific IKK inhibitor BMS-345541 [34] inhibited xFANCD2-Ub in a manner similar to EF24, i.e. xFANCD2-Ub inhibition occurred at higher concentration than IkB- $\alpha$ stabilization (Fig 3A, B). This differential effect is reminiscent of the fact that BMS-345541 is 10fold more active on IKK-2 (IKK $\beta$ ) than on IKK-1 (IKK $\alpha$ ), maybe because allosteric binding induces a different conformational change in the two IKK subunits [34]. The latter result also suggests that the kinase activity of IKK is not required for plasmid activation of the FA pathway in extracts. The fact that general phosphorylation modulators did not interfere with EF24 (Fig. 3C) provided an indirect confirmation of this hypothesis. Additional experiments are needed to explore the potential link between EF24, IKK and the FA pathway.

Like curcumin, the mimics depicted in Figure 1 are pleiotropic agents with activities as diverse as mitochondrial redox-mediators [19], tubulin dynamics disrupters [22], kinase inhibitors [32], glioma cell radiosensitizers [48] and angiogenesis blockers [21]. Although their multiplicity of actions is complex, predictive pharmacological models [49] and optimization [50] are emerging within systems biology. The discovery that monoketone analogs of curcumin form a new class of FA pathway inhibitors not only underscores their potential for low toxicity targeted cancer therapy, it also adds to the still incomplete network of beneficial biological actions exerted by these multi-tasking agents.

\section{Conclusions}

Using curcumin as a lead we identified EF24, a monoketone analog with improved activity and specificity toward the FA pathway. An independent screen identified $4 \mathrm{H}$ TTD, a structurally related compound with similar activities, suggesting that monoketone analogs form a new class of FA pathway inhibitors. Fig. 8 summarizes the main mechanistic findings of the study.

To our knowledge, this is the first report suggesting that small molecule inhibition of the FA pathway may specifically target ATM-deficient cells. Additional experiments are under way to confirm this synthetic lethal effect and to test whether these compounds are effective in human ATM-deficient cancer cells as well.

\section{Methods}

\section{Chemicals and antibodies}

Monoketone analogs of curcumin were prepared as previously described [18]. Pure curcumin, MG132 (Calbiochem), IKK inhibitor III (BMS-345541, Calbiochem), DRB (Calbiochem), 4H-TTD (NSC 144303, NCI/DTP Open Chemical Repository, http://dtp.nci.nih.gov) were resuspended in DMSO, tautomycin (Calbiochem) in EtOH, caffeine and hydroxyurea (Sigma) in $\mathrm{H}_{2} \mathrm{O}$. Shrimp alkaline phosphatase was from Fermentas. Antibodies against xFANCD2, xFANCG, xFANCA and hFANCD2 were described previously [6]. Antibodies against $\gamma \mathrm{H} 2 \mathrm{AX}$ and IkB- $\alpha$ were from Bethyl laboratories (\#A300-081A) and cell Signaling (\#9242), respectively. Antibodies against xRPA, xMre11 and hFANCD2 (for immunofluorescence) were kind gifts of K. Cimprich, J. Gautier and K.J. Patel, respectively.

\section{Xenopus cell-free assay and immunoblotting}

Preparation of Xenopus laevis low-speed extracts and the FA pathway assay were described previously [6].

\section{Proteasome assay}

Fluorogenic peptides (Proteasome Substrate Pack \#PW9905) specific for the chymotrypsin-like and caspaselike activities of the proteasome were incubated in Xenopus extracts treated with serial dilutions of various compounds. Fluorescence emitted by proteasome cleavage of the peptides was monitored using a fluorometer (FluoStar Galaxy, BMG Labtech) with $380 \mathrm{~nm}$ and $460 \mathrm{~nm}$ excitation and emission filters, respectively. Experiments were repeated at least twice. Due to the large variability of activity between extract batches, one representative experiment is shown.

\section{Cell lines and cell culture}

HeLa cells were grown in DMEM medium supplemented with 10\% serum in humidified 5\% CO2 atmosphere. ATM-deficient and ATM-proficient kidney cells from con- 


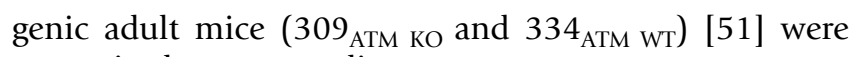
grown in the same medium.

\section{hFANCD2 ubiquitylation assay and immunofluorescence microscopy in HeLa cells}

hFANCD2 ubiquitylation assay and immunofluorescence assays in HeLa cells have been described elsewhere [6].

\section{Survival assays}

HSC72OT and HSC72OT+A cells were seeded at 5,000 per well in 96-well plates, and treated 24 hrs later with various concentrations of compounds or DMSO $(1 \mu \mathrm{l}$ in 100 $\mu \mathrm{l}$ medium). $309_{\text {ATM KO }}$ and 334 $4_{\text {ATM WT }}$ cells were seeded at 7,000 per well and treated immediately. Cell survival was measured after 3 days using the CellTiter 96 AQueous One Solution Cell Proliferation Assay (Promega) following the recommendations from the manufacturer. All experiments were done in triplicate.

\section{Calculation of $I C_{50}$ values}

Fitted curves were determined from experimental data using IgorPro. The concentration of compound that induces 50\% inhibition of the measured endpoint (i.e. FANCD2 ubiquitylation, Mre11 phosphorylation and cell survival) was then plotted on the fitted curve.

\section{Competing interests}

The authors declare that they have no competing interests.

\section{Authors' contributions}

IL designed the study, performed experiments in Xenopus extracts, immunofluorescence, FACS analysis and drafted the manuscript. SH performed cell-based hFANCD2 ubiquitylation assays and cell survival assays. MMC performed cell survival assays. AS prepared curcumin analogs in Fig. 1 and helped draft the manuscript. CY prepared fresh samples of 4H-TTD. MST prepared ATM WT and KO cells and helped draft the manuscript. JPS chose the analogs for chemical testing and wrote the chemical section. MEH conceived the study, coordinated lab efforts and helped draft the manuscript. All authors read and approved the final manuscript.

\section{Additional material}

\section{Additional file 1}

Supplementary Figures. This file contains four supplementary figures. Figure S1: EF24 does not inhibit the FA pathway through disruption of the core complex in Xenopus extracts. Figure S2: Combination of EF24 with $\mathrm{HU}$ does not significantly alter the cell cycle compared to untreated cells. Figure S3: $309_{A T M ~ K O}$ cells are more sensitive to ionizing irradiation than $334_{\text {ATM WT }}$ cells. Figure S4: EF24 inhibits phosphorylation of CHK1 (CHK1-P) in DNA-stimulated Xenopus extracts.

Click here for file

[http://www.biomedcentral.com/content/supplementary/14764598-8-133-S1.PDF]

\section{Acknowledgements}

We thank Dr. K. Cimprich, Dr. J. Gautier and Dr K.J. Patel for generously sharing antibodies for xRPA, xMrel I and hFANCD2, respectively. We are grateful to Marion Lanier, from the Human BioMolecular Research Institute, for chemical structure analysis and to Professor Dennis Liotta (Emory University) for encouragement and support. Financial support: M. Hoatlin (NIH CA I I 2775, OHSU BioScience Innovation Fund Grant and the Fanconi Anemia Research Fund (FARF); I. Landais (NRSA postdoctoral training grant CA 101690-03).

\section{References}

I. Kalb R, Neveling K, Nanda I, Schindler D, Hoehn H: Fanconi anemia: causes and consequences of genetic instability. Genome Dyn 2006, I:2 18-242.

2. de Winter JP, Joenje H: The genetic and molecular basis of Fanconi anemia. Mutat Res 2008.

3. Wang W: Emergence of a DNA-damage response network consisting of Fanconi anaemia and BRCA proteins. Nat Rev Genet 2007, 8:735-748.

4. Rahman N, Scott RH: Cancer genes associated with phenotypes in monoallelic and biallelic mutation carriers: new lessons from old players. Hum Mol Genet 2007, I 6(Spec No I):R60-66.

5. Chirnomas D, Taniguchi T, de la Vega M, Vaidya AP, Vasserman M, Hartman AR, Kennedy R, Foster R, Mahoney J, Seiden MV, D'Andrea $A D$ : Chemosensitization to cisplatin by inhibitors of the Fanconi anemia/BRCA pathway. Mol Cancer Ther 2006, 5:952-961.

6. Landais I, Sobeck A, Stone S, LaChapelle A, Hoatlin ME: A novel cellfree screen identifies a potent inhibitor of the Fanconi anemia pathway. Int / Cancer 2009, I 24:783-792.

7. Ashworth A: A synthetic lethal therapeutic approach: poly(ADP) ribose polymerase inhibitors for the treatment of cancers deficient in DNA double-strand break repair. J Clin Oncol 2008, 26:3785-3790.

8. Fong PC, Boss DS, Yap TA, Tutt A, Wu P, Mergui-Roelvink M, Mortimer $\mathrm{P}$, Swaisland $\mathrm{H}$, Lau A, O'Connor MJ, et al:: Inhibition of poly(ADP-ribose) polymerase in tumors from BRCA mutation carriers. N Engl J Med 2009, 36 I: | 23-134.

9. Tuma RS: PARP inhibitors: will the new class of drugs match the hype? J Natl Cancer Inst 2009, I0 I: I230-1 232.

10. Scholl C, Frohling S, Dunn IF, Schinzel AC, Barbie DA, Kim SY, Silver SJ, Tamayo P, Wadlow RC, Ramaswamy S, et al.: Synthetic lethal interaction between oncogenic KRAS dependency and STK33 suppression in human cancer cells. Cell 2009, 137:821-834.

II. Luo J, Emanuele MJ, Li D, Creighton CJ, Schlabach MR, Westbrook TF, Wong KK, Elledge SJ: A genome-wide RNAi screen identifies multiple synthetic lethal interactions with the Ras oncogene. Cell 2009, 137:835-848.

12. Kennedy RD, Chen CC, Stuckert P, Archila EM, De la Vega MA, Moreau LA, Shimamura A, D'Andrea AD: Fanconi anemia pathway-deficient tumor cells are hypersensitive to inhibition of ataxia telangiectasia mutated. J Clin Invest 2007, I I 7: I 440- I 449.

13. Czornak K, Chughtai S, Chrzanowska KH: Mystery of DNA repair: the role of the MRN complex and ATM kinase in DNA damage repair. J Appl Genet 2008, 49:383-396.

14. Lavin MF: Ataxia-telangiectasia: from a rare disorder to a paradigm for cell signalling and cancer. Nat Rev Mol Cell Biol 2008, 9:759-769.

15. Austen B, Skowronska A, Baker C, Powell JE, Gardiner A, Oscier D, Majid A, Dyer M, Siebert R, Taylor AM, et al.: Mutation status of the residual ATM allele is an important determinant of the cellular response to chemotherapy and survival in patients with chronic lymphocytic leukemia containing an IIq deletion. J Clin Oncol 2007, 25:5448-5457.

16. Fang NY, Greiner TC, Weisenburger DD, Chan WC, Vose JM, Smith LM, Armitage JO, Mayer RA, Pike BL, Collins FS, Hacia JG: Oligonucleotide microarrays demonstrate the highest frequency of ATM mutations in the mantle cell subtype of lymphoma. Proc Natl Acad Sci USA 2003, 100:5372-5377.

17. Aggarwal BB, Sundaram C, Malani N, Ichikawa H: Curcumin: the Indian solid gold. Adv Exp Med Biol 2007, 595: I-75.

18. Adams BK, Ferstl EM, Davis MC, Herold M, Kurtkaya S, Camalier RF, Hollingshead MG, Kaur G, Sausville EA, Rickles FR, et al.: Synthesis and biological evaluation of novel curcumin analogs as anti- 
cancer and anti-angiogenesis agents. Bioorg Med Chem 2004 I 2:387|-3883

19. Adams BK, Cai J, Armstrong J, Herold M, Lu YJ, Sun A, Snyder JP, Liotta DC, Jones DP, Shoji M: EF24, a novel synthetic curcumin analog, induces apoptosis in cancer cells via a redox-dependent mechanism. Anticancer Drugs 2005, 16:263-275.

20. Sun A, Shoji M, Lu YJ, Liotta DC, Snyder JP: Synthesis of EF24tripeptide chloromethyl ketone: a novel curcumin-related anticancer drug delivery system. J Med Chem 2006 49:3।53-3।58.

21. Shoji M, Sun A, Kisiel W, Lu YJ, Shim H, McCarey BE, Nichols C, Parker ET, Pohl J, Mosley CA, et al:: Targeting tissue factorexpressing tumor angiogenesis and tumors with EF24 conjugated to factor VIla. J Drug Target 2008, 16:185-197.

22. Thomas SL, Zhong D, Zhou W, Malik S, Liotta D, Snyder JP, Hamel E, Giannakakou P: EF24, a novel curcumin analog, disrupts the microtubule cytoskeleton and inhibits HIF-I. Cell Cycle 2008, 7:2409-24I7.

23. Sobeck A, Stone S, Costanzo V, de Graaf B, Reuter T, de Winter J, Wallisch M, Akkari Y, Olson S, Wang W, et al.: Fanconi anemia proteins are required to prevent accumulation of replication-associated DNA double-strand breaks. Mol Cell Biol 2006, 26:425-437.

24. Sobeck $A$, Stone $S$, Hoatlin ME: DNA structure-induced recruitment and activation of the Fanconi anemia pathway protein FANCD2. Mol Cell Biol 2007, 27:4283-4292.

25. Ben-Yehoyada M, Wang LC, Kozekov ID, Rizzo CJ, Gottesman ME, Gautier J: Checkpoint signaling from a single DNA interstrand crosslink. Mol Cell 2009, 35:704-7I5.

26. Wang LC, Stone S, Hoatlin ME, Gautier J: Fanconi anemia proteins stabilize replication forks. DNA Repair (Amst) 2008, 7:1973-1981.

27. Costanzo V, Robertson K, Bibikova M, Kim E, Grieco D, Gottesman M, Carroll D, Gautier J: Mre I I protein complex prevents double-strand break accumulation during chromosomal DNA replication. Mol Cell 200I, 8: I37-I47.

28. Di Virgilio M, Ying CY, Gautier J: PIKK-dependent phosphorylation of Mrell induces MRN complex inactivation by disassembly from chromatin. DNA Repair (Amst) 2009.

29. Lupardus PJ, Byun T, Yee MC, Hekmat-Nejad M, Cimprich KA: A requirement for replication in activation of the ATRdependent DNA damage checkpoint. Genes Dev 2002, 16:2327-2332

30. Jacquemont $C$, Taniguchi $T$ : Proteasome function is required for DNA damage response and fanconi anemia pathway activation. Cancer Res 2007, 67:7395-7405.

31. Jana NR, Dikshit P, Goswami A, Nukina N: Inhibition of proteasomal function by curcumin induces apoptosis through mitochondrial pathway. J Biol Chem 2004, 279: I I680-I I685.

32. Kasinski AL, Du Y, Thomas SL, Zhao J, Sun SY, Khuri FR, Wang CY, Shoji M, Sun A, Snyder JP, et al.: Inhibition of IkappaB kinasenuclear factor-kappaB signaling pathway by 3,5-bis(2flurobenzylidene)piperidin-4-one (EF24), a novel monoketone analog of curcumin. Mol Pharmacol 2008, 74:654-66I.

33. Otsuki T, Young DB, Sasaki DT, Pando MP, Li J, Manning A, Hoekstra $M$, Hoatlin ME, Mercurio F, Liu JM: Fanconi anemia protein complex is a novel target of the IKK signalsome. J Cell Biochem 2002, 86:613-623.

34. Burke JR, Pattoli MA, Gregor KR, Brassil PJ, MacMaster JF, Mclntyre KW, Yang X, lotzova VS, Clarke W, Strnad J, et al.: BMS-34554I is a highly selective inhibitor of I kappa $B$ kinase that binds at an allosteric site of the enzyme and blocks NF-kappa Bdependent transcription in mice. I Biol Chem 2003, 278: $1450-\mid 456$.

35. Taniguchi T, Garcia-Higuera I, Andreassen PR, Gregory RC, Grompe $M$, D'Andrea AD: S-phase-specific interaction of the Fanconi anemia protein, FANCD2, with BRCAI and RAD5I. Blood 2002, 100:24|4-2420.

36. Duckworth-Rysiecki G, Cornish K, Clarke CA, Buchwald M: Identification of two complementation groups in Fanconi anemia. Somat Cell Mol Genet 1985, I I:35-4I.

37. Rai R, Peng G, Li K, Lin SY: DNA damage response: the players, the network and the role in tumor suppression. Cancer Genomics Proteomics 2007, 4:99-106.
38. Wang ZO, Stingl L, Morrison C, Jantsch M, Los M, Schulze-Osthoff K, Wagner EF: PARP is important for genomic stability but dispensable in apoptosis. Genes Dev 1997, I I:2347-2358.

39. Ak T, Gulcin I: Antioxidant and radical scavenging properties of curcumin. Chem Biol Interact 2008, 174:27-37.

40. Patro BS, Rele S, Chintalwar G], Chattopadhyay S, Adhikari S, Mukherjee T: Protective activities of some phenolic I,3-diketones against lipid peroxidation: possible involvement of the I,3-diketone moiety. Chembiochem 2002, 3:364-370.

4I. Dupre A, Boyer-Chatenet L, Sattler RM, Modi AP, Lee JH, Nicolette ML, Kopelovich L, Jasin M, Baer R, Paull TT, Gautier J: A forward chemical genetic screen reveals an inhibitor of the Mre IIRad50-NbsI complex. Nat Chem Biol 2008, 4: I I9-125.

42. Gurtan AM, Stuckert P, D'Andrea AD: The WD40 repeats of FANCL are required for Fanconi anemia core complex assembly. J Biol Chem 2006, 28 I: 10896-10905.

43. Aleo E, Henderson C], Fontanini A, Solazzo B, Brancolini C: Identification of new compounds that trigger apoptosome-independent caspase activation and apoptosis. Cancer Res 2006, 66:9235-9244.

44. Sobeck A, Stone S, Landais I, de Graaf B, Hoatlin ME: The Fanconi anemia protein FANCM is controlled by FANCD2 and the ATR/ATM pathways. J Biol Chem 2009, 284:25560-25568.

45. Liu Q, Guntuku S, Cui XS, Matsuoka S, Cortez D, Tamai K, Luo G, Carattini-Rivera S, DeMayo F, Bradley A, et al.: Chk I is an essential kinase that is regulated by Atr and required for the $G(2) / M$ DNA damage checkpoint. Genes Dev 2000, I 4: I 448- 4459.

46. Ho GP, Margossian S, Taniguchi T, D'Andrea AD: Phosphorylation of FANCD2 on two novel sites is required for mitomycin C resistance. Mol Cell Biol 2006, 26:7005-70I5.

47. Ishiai M, Kitao H, Smogorzewska A, Tomida J, Kinomura A, Uchida E, Saberi A, Kinoshita E, Kinoshita-Kikuta E, Koike T, et al.: FANCI phosphorylation functions as a molecular switch to turn on the Fanconi anemia pathway. Nat Struct Mol Biol 2008, I5: I |38-I I 46.

48. Shu HG, Brown A, Yoon Y, Gao H, Purcell J, Snyder JP, Liotta DC, Shim $\mathrm{H}$ : Radiosensitization of glioma cells with the curcumin analogs EF24 and UBS 109. AACR 99th Annual Meeting 2008.

49. Keiser MJ, Roth BL, Armbruster BN, Ernsberger P, Irwin J], Shoichet BK: Relating protein pharmacology by ligand chemistry. Nat Biotechnol 2007, 25:197-206.

50. Yang K, Bai H, Ouyang Q, Lai L, Tang C: Finding multiple target optimal intervention in disease-related molecular network. Mol Syst Biol 2008, 4:228.

51. Gage BM, Alroy D, Shin CY, Ponomareva ON, Dhar S, Sharma GG, Pandita TK, Thayer MJ, Turker MS: Spontaneously immortalized cell lines obtained from adult Atm null mice retain sensitivity to ionizing radiation and exhibit a mutational pattern suggestive of oxidative stress. Oncogene 200I, 20:429I-4297.
Publish with BioMed Central and every scientist can read your work free of charge

"BioMed Central will be the most significant development for disseminating the results of biomedical research in our lifetime. "

Sir Paul Nurse, Cancer Research UK

Your research papers will be:

- available free of charge to the entire biomedical community

- peer reviewed and published immediately upon acceptance

- cited in PubMed and archived on PubMed Central

- yours - you keep the copyright
BioMedcentral 